\title{
Observations of extended very-high-energy halos around Geminga and Monogem with the LHAASO-KM2A
}

\author{
Yingying Guo, ${ }^{a, b}, *$ Yi Zhang, ${ }^{b}$ Qiang Yuan ${ }^{b}$ and Hongbo $\mathrm{Hu}^{a}$ on behalf of the \\ LHAASO collabration Collaboration \\ (a complete list of authors can be found at the end of the proceedings) \\ ${ }^{a}$ Institute of High Energy Physics, Chinese Academy of Sciences \\ 100049 Beijing, China \\ ${ }^{b}$ Purple Mountain Observatory, Chinese Academy of Sciences \\ 210023 Nanjing, Jiangsu, China \\ E-mail: yyguo@pmo.ac.cn
}

Gamma-ray halos around pulsars are an effective probe of particle propagation in the interstellar medium. Two halos named Geminga and Monogem are observed by the KM2A, one of the sub arrays of the Large High Altitude Air Shower Observatory (LHAASO). The significance of Geminga(Monogem) is $11 \sigma(7 \sigma)$ at the energy range from $25 \mathrm{TeV}$ to $63 \mathrm{TeV}$. In the future, more statistics will allow us to investigate the energy-dependent morphologies.

$37^{\text {th }}$ International Cosmic Ray Conference (ICRC 2021)

July 12 th - 23rd, 2021

Online - Berlin, Germany

\footnotetext{
${ }^{*}$ Presenter
} 


\section{Introduction}

The origin and propagation of cosmic rays (CRs) are very important questions in physics and astrophysics. The conventional model suggests that CRs, after being accelerated by extreme astronomical objects, propagate diffusively in the random magnetic field of the Milky Way, interact with matter and field during the propagation and produce secondary particles [2]. The diffusion coefficient is the most important parameter to characterize the propagation of CRs. It is also a very important measure of the property of the interstellar medium. Usually one can use the secondary-toprimary ratio of $\mathrm{CR}$ nuclei, such as the Boron-to-Carbon ratio, to measure the diffusion coefficient.

Observations of ultra-high-energy (UHE) gamma rays are another very important method to probe the origin and propagation of CRs. In 2017, the High Altitude Water Cherenkov observatory (HAWC) discovered extended very-high-energy (VHE) gamma-ray halos around two middle-aged pulsars, Geminga with an age of $342 \mathrm{kyr}$ and Monogem with an age of $110 \mathrm{kyr}$, which are expected to be produced by UHE electrons and positrons accelerated by the pulsars and then radiate through inverse Compton scattering off the interstellar radiation field [1]. Through measuring the morphologies of the extended emission, the HAWC collaboration concluded that charged particles diffuse extremely slowly around those pulsars. The diffusion coefficient inferred from the HAWC gamma-ray observations is smaller by two orders of magnitude than that derived from the CR Boron-to-Carbon ratio.

However, the energy-dependence of the diffusion coefficient around Geminga remains a puzzle, which is a crucial key to investigate the properties of magnetic field turbulence. Because the diffusion coefficient is determined by the average strength of the magnetic field B and its degree of turbulence $\delta B$ on length scales comparable to the gyration radius. Traditionally, the diffusion coefficient grows with energy according to a power law, the exponent of which depends on the turbulent nature of the magnetic field. In Kolmogorov's theory, the exponent equals 1/3 power[4]; in Kraichnan's hypothesis, the exponent is $1 / 2$ power[5]; in the Bohm Limit case, the exponent equals 1 power.

LHAASO-KM2A is the most sensitive observatory for gamma rays above $20 \mathrm{TeV}$ and is thus a very powerful probe of the UHE gamma-ray emitter. Compared with HAWC, LHAASO is superior in the energy band coverage, energy resolution, and sensitivity. LHAASO has a large field of view and is therefore very appropriate for the extended source observations. LHAASO is unique to reveal the energy-dependent morphologies of the emission, and can provide very important information on the energy-dependence of the diffusion coefficient.

\section{LHAASO-KM2A}

The kilometer square array(KM2A) is an important part of LHAASO that is located at Haizi Mountain, Daocheng, Sichuan province, China. It is currently the most sensitive detector above $20 \mathrm{TeV}$ and the half array of LHAASO-KM2A (1/2KM2A) has been continuously observing VHE gamma rays since 2019. As shown in the figure 1, 1/2KM2A contains 2365 scintillator detectors (Electromagnetic particle Detector, ED) and 578 muon detectors (MD), where the EDs are used to reconstruct the direction of the event and the MDs mainly detect the Muon component of secondary particles, which are used to distinguish between gamma and proton, since the Muons produced by gamma rays is poorer than those from protons. The rejection power of cosmic ray induced 
showers is better than $4 \times 10^{3}$ at energies above $100 \mathrm{TeV}$. The $1 / 2 \mathrm{KM} 2 \mathrm{~A}$ data collected from 27 th December,2019 to 7th December, 2020 is used in this analysis.

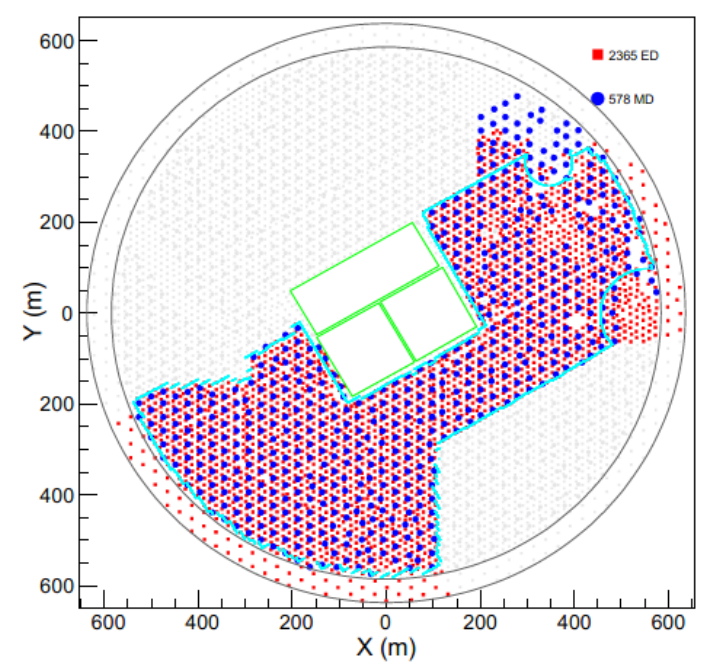

Figure 1: Layout of the whole LHAASO-KM2A.The red circles and blue squares indicate the EDs and MDs in operation, respectively. The light gray markers indicate the prospective EDs and MDs.

The simulation sample used in this study is the same as in the Crab analysis, and the simulated samples of both sources were produced by reweighting the event according to the source tracks. Its reliability has been verified in the paper [3].

\section{3. method}

To estimate the background mainly contributed from cosmic rays, the equi-zenith angle method is adopted. This method assumes that detector effectiveness is the same throughout zenith belts and the background of one grid can be valued as the average of the sideband. In addition, we mask the Galactic Plane and other known $\mathrm{TeV}$-source regions, in case that the gamma-ray sources are at the sideband and overestimate the background. The mask region for the Geminga and Monogem is two circles with a radius of 15 degrees due to their large extension.

The maximum likelihood ratio is constructed by comparing the diffusion model with the none source model to analyze the morphologies of both sources. The diffusion template read as formula 1 , where $\mathrm{A}$ is the normalization constant indicating the signal intensity, $\theta_{d}$ is the diffusion angle, $\theta_{d}=\frac{180^{\circ}}{\pi} \cdot \frac{2 \sqrt{D\left(E_{e}\right) t_{E}}}{r}$, and $t_{E}$ is the cooling time of the electron, $\mathrm{r}$ is the source distance. This template describes gamma rays produced by continuously injecting electrons via Inverse Compton(IC) progress. To avoid the detector impact on the $\theta_{d}$, the convolution function of diffusion template and angular resolution is used to fit experimental data. Due to the similarity of both sources in age, we presume that the diffusion property is the same for both sources. To avoid contamination from other known sources, we deducted a circular area of 2 degrees around each source, as shown in Fig. 2.

$$
f(\theta)=\frac{A}{\theta_{d}\left(\theta+0.085 \theta_{d}\right)} \exp \left[-1.54\left(\theta / \theta_{d}\right)^{1.52}\right],
$$




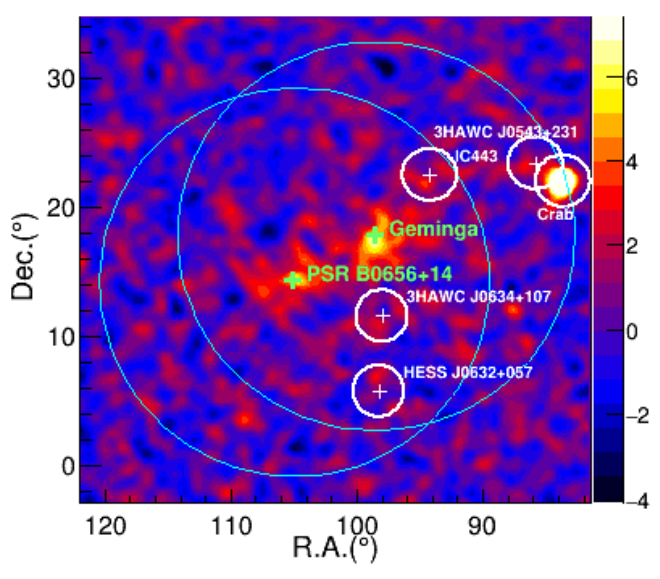

Figure 2: The figure shows the significance of Geminga in the range of $15-25 \mathrm{TeV}$. The blue circle with a radius of 15 degrees indicates the region of intrerest. the white circle with a radius of two degrees in the figure indicates the deducted region of known intrahelial sources.

\section{Results and discussion}

The clear excesses around Geminga and Monogem in different energy ranges are observed in Fig.3. The two sources are marked as full crosses and the statistical significances of Geminga(Monogem) are $8 \sigma(7 \sigma)$ at $10-25,11 \sigma(7 \sigma)$ at $25-63 \mathrm{TeV}$. This map is obtained by the likelihood test between the two-dimensional Gaussian model and the background-only model, the Gaussian sigma values are $0.5^{\circ}, 0.26^{\circ}$ and $0.25^{\circ}$, which corresponds to the PSF of KM2A in each energy range.
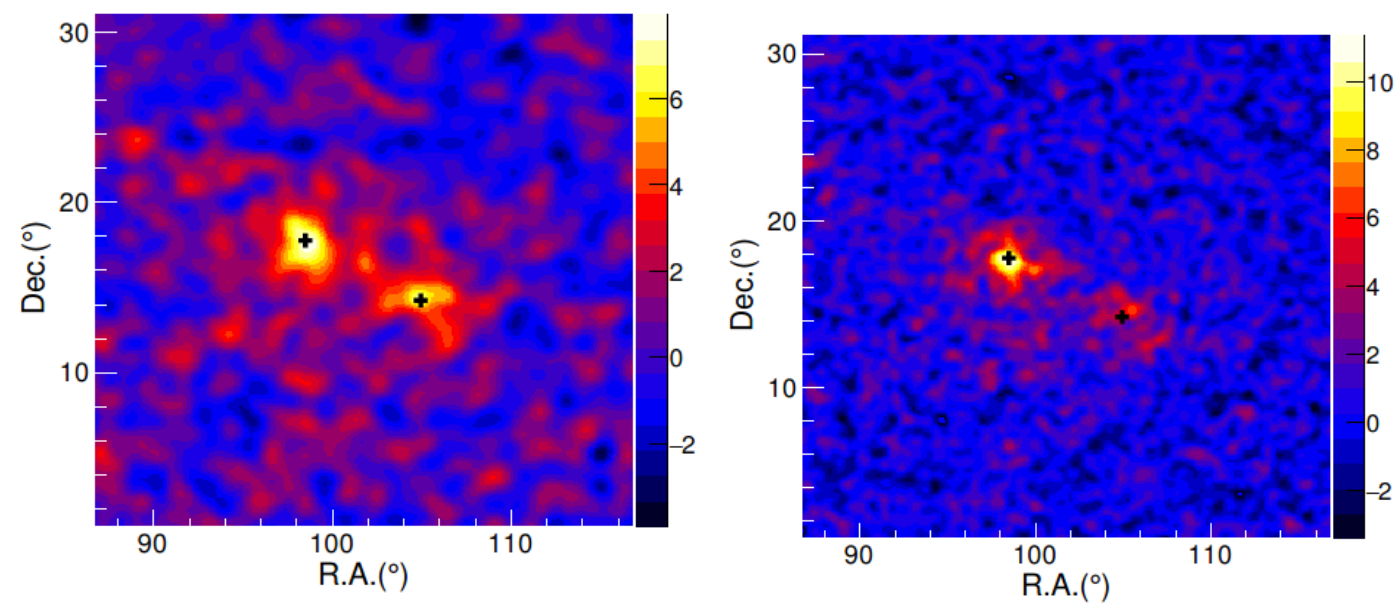

Figure 3: Significance maps of Geminga and Monogem at different energies(left: $15 \mathrm{TeV}<\mathrm{E}<25 \mathrm{TeV}$ Right: $25 \mathrm{TeV}<\mathrm{E}<63 \mathrm{TeV}$ ). The two black crosses denote the Geminga Pulsar(upper left) and the Monogem Pulsar(bottom right) respectively.

The morphology analysis is going on and more data by KM2A in the future will allow to investigate the energy-dependence of the morphology. 


\subsection{Acknowledgements}

This work is supported in China by the National Key R\&D program of China under the grants 2018YFA0404202, by the National Natural Science Foundation of China under the grants 11775233.

\section{References}

[1] Abeysekara A U, Albert A, Alfaro R, et al. Science, 2017, 358(6365): 911-914.DOI: $10.1126 /$ science.aan 4880 .

[2] Ginzburg V L, Syrovatskii S I. The Origin of Cosmic Rays [M]. 1964.

[3] Aharonian, F., An, Q., Axikegu, et al. 2020, Chin. Phys. C, 4025002,arXiv:2010.06205

[4] Osonphasop, C.,Nakagawa, T. 2014, Open Journal of Fluid Dynamics, 4: 140153.DOI:10.4236/ojfd.2014.42013

[5] Robert H. Kraichnan. 1965, The Physics of Fluids 8, 8(7):1385-1387.DOI:10.1063/1.1761412 


\section{Full Authors List: LHAASO collabration Collaboration}

Zhen $\mathrm{Cao}^{1,2,3}$, F. Aharonian ${ }^{4,5}$, Q. An ${ }^{6,7}$, Axikegu ${ }^{8}$, L.X. Bai ${ }^{9}$, Y.X. Bai ${ }^{1,3}$, L.X. Bai ${ }^{9}$, Y.X. Bai ${ }^{1,3}$, Y.W. Bao ${ }^{10}$, D. Bastieri ${ }^{11}$, X.J. Bi $1^{1,2,3}$, Y.J. Bi ${ }^{1,3}$, H. Cai ${ }^{12}$, J.T. Cai ${ }^{11}$, Zhe Cao ${ }^{6,7}$, J. Chang ${ }^{13}$, J.F. Chang ${ }^{1,3,6}$, B.M. Chen ${ }^{14}$, E.S. Chen ${ }^{1,2,3}$, J. Chen ${ }^{9}$, Liang Chen $^{1,2,3}$, Liang Chen ${ }^{15}$, Long Chen ${ }^{8}$, M.J. Chen ${ }^{1,3}$, M.L. Chen ${ }^{1,3,6}$, Q.H. Chen ${ }^{8}$, S.H. Chen ${ }^{1,2,3}$, S.Z. Chen ${ }^{1,3}$, T.L. Chen ${ }^{16}$,X.L. Chen $^{1,2,3}$, Y. Chen ${ }^{10}$, N. Cheng ${ }^{1,3}$, Y.D. Cheng ${ }^{1,3}$, S.W. Cui ${ }^{14}$, X.H. Cui ${ }^{17}$, Y.D. Cui ${ }^{18}$, B. D'Ettorre Piazzoli ${ }^{19}$, B.Z. Dai ${ }^{20}$, H.L. Dai $^{1,3,6}$, Z.G. Dai ${ }^{7}$, Danzengluobu ${ }^{16}$, D. della Volpe ${ }^{21}$, X.J. Dong ${ }^{1,3}$, K.K. Duan ${ }^{13}$, J.H. Fan ${ }^{11}$, Y.Z. Fan ${ }^{13}$, Z.X. Fan ${ }^{1,3}$, J. Fang ${ }^{20}$, K. Fang $^{1,3}$, C.F. Feng ${ }^{22}$, L. Feng ${ }^{13}$, S.H. Feng ${ }^{1,3}$, Y.L. Feng ${ }^{13}$, B. Gao ${ }^{1,3}$, C.D. Gao ${ }^{22}$, L.Q. Gao ${ }^{1,2,3}$, Q. Gao ${ }^{16}$, W. Gao ${ }^{22}$, M.M. Ge Ge $^{20}$, L.S. Geng ${ }^{1,3}$, G.H. Gong ${ }^{23}$, Q.B. Gou ${ }^{1,3}$, M.H. Gu${ }^{1,3,6}$, F.L. Guo ${ }^{15}$, J.G. Guo ${ }^{1,2,3}$, X.L. Guo ${ }^{8}$, Y.Q. Guo ${ }^{1,3}$, Y.Y. Guo ${ }^{1,2,3,13}$, Y.A. $\mathrm{Han}^{24}$, H.H. He ${ }^{1,2,3}$, H.N. He ${ }^{13}$, J.C. He ${ }^{1,2,3}$, S.L. He ${ }^{11}$, X.B. He ${ }^{18}$, Y. He ${ }^{8}$, M. Heller ${ }^{21}$, Y.K. Hor ${ }^{18}$, C. Hou ${ }^{1,3}$, H.B. Hu ${ }^{1,2,3}$, S. $\mathrm{Hu}^{9}$, S.C. $\mathrm{Hu}^{1,2,3}$, X.J. $\mathrm{Hu}^{23}$, D.H. Huang ${ }^{8}$, Q.L. Huang ${ }^{1,3}$, W.H. Huang ${ }^{22}$, X.T. Huang ${ }^{22}$, X.Y. Huang ${ }^{13}$, Z.C. Huang ${ }^{8}$, F. Ji ${ }^{1,3}$, X.L. $\mathrm{Ji}^{1,3,6}$, H.Y. Jia ${ }^{8}$, K. Jiang ${ }^{6,7}$, Z.J. Jiang ${ }^{20}$, C. Jin ${ }^{1,2,3}$, T. Ke ${ }^{1,3}$, D. Kuleshov ${ }^{25}$, K. Levochkin ${ }^{25}$, B.B. Li ${ }^{14}$, Cheng Li ${ }^{6,7}, \mathrm{Cong} \mathrm{Li}^{1,3}$,

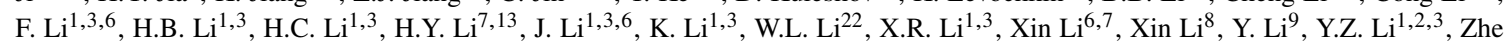

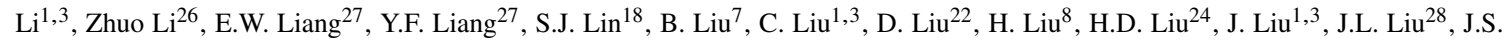

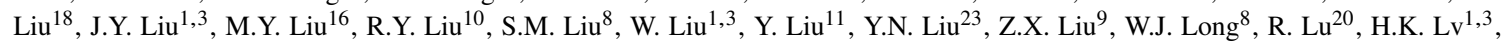

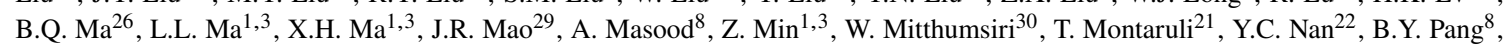
P. Pattarakijwanich ${ }^{30}$, Z.Y. Pei ${ }^{11}$, M.Y. Qi ${ }^{1,3}$, Y.Q. Qi ${ }^{14}$, B.Q. Qiao ${ }^{1,3}$, J.J. Qin ${ }^{7}$, D. Ruffolo ${ }^{30}$, V. Rulev ${ }^{25}$, A. Sáiz ${ }^{30}$, L. Shao ${ }^{14}$, O. Shchegolev $^{25,31}$, X.D. Sheng ${ }^{1,3}$, J.Y. Shi ${ }^{1,3}$, H.C. Song ${ }^{26}$, Yu.V. Stenkin ${ }^{25,31}$, V. Stepanov ${ }^{25}$, Y. Su ${ }^{32}$, Q.N. Sun ${ }^{8}$, X.N. Sun ${ }^{27}$, Z.B.

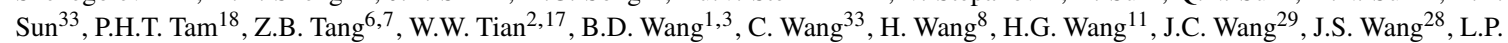
Wang $^{22}$, L.Y. Wang ${ }^{1,3}$, R.N. Wang ${ }^{8}$, W. Wang ${ }^{18}$, W. Wang ${ }^{12}$, X.G. Wang ${ }^{27}$, X.J. Wang ${ }^{1,3}$, X.Y. Wang ${ }^{10}$, Y. Wang ${ }^{8}$, Y.D. Wang ${ }^{1,3}$, Y.J.

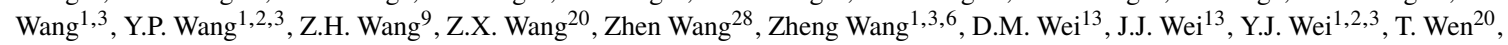
C.Y. Wu ${ }^{1,3}$, H.R. Wu ${ }^{1,3}$, S. Wu ${ }^{1,3}$, W.X. Wu ${ }^{8}$, X.F. Wu ${ }^{13}$, S.Q. Xi ${ }^{1,3}$, J. Xia ${ }^{7,13}$, J.J. Xia ${ }^{8}$, G.M. Xiang ${ }^{2,15}$, D.X. Xiao ${ }^{16}$, G. Xiao ${ }^{1,3}$, H.B. Xiao ${ }^{11}$, G.G. Xin ${ }^{12}$, Y.L. Xin ${ }^{8}$, Y. Xing ${ }^{15}$, D.L. Xu ${ }^{28}$, R.X. Xu ${ }^{26}$, L. Xue ${ }^{22}$, D.H. Yan ${ }^{29}$, J.Z. Yan ${ }^{13}$, C.W. Yang ${ }^{9}$, F.F. Yang ${ }^{1,3,6}$, J.Y. Yang ${ }^{18}$, L.L. Yang ${ }^{18}$, M.J. Yang ${ }^{1,3}$, R.Z. Yang ${ }^{7}$, S.B. Yang ${ }^{20}$, Y.H. Yao ${ }^{9}$, Z.G. Yao ${ }^{1,3}$, Y.M. Ye ${ }^{23}$, L.Q. Yin ${ }^{1,3}$, N. Yin ${ }^{22}$, X.H. You $^{1,3}$, Z.Y. You ${ }^{1,2,3}$, Y.H. Yu ${ }^{22}$, Q. Yuan ${ }^{13}$, H.D. Zeng ${ }^{13}$, T.X. Zeng ${ }^{1,3,6}$, W. Zeng ${ }^{20}$, Z.K. Zeng ${ }^{1,2,3}$, M. Zha ${ }^{1,3}$, X.X. Zhai ${ }^{1,3}$, B.B. Zhang ${ }^{10}$, H.M. Zhang ${ }^{10}$, H.Y. Zhang ${ }^{22}$, J.L. Zhang ${ }^{17}$, J.W. Zhang ${ }^{9}$, L.X. Zhang ${ }^{11}$, Li Zhang ${ }^{20}$, Lu Zhang ${ }^{14}$, P.F. Zhang ${ }^{20}$, P.P. Zhang ${ }^{14}$, R. Zhang ${ }^{7,13}$, S.R. Zhang ${ }^{14}$, S.S. Zhang ${ }^{1,3}$, X. Zhang ${ }^{10}$, X.P. Zhang ${ }^{1,3}$, Y.F. Zhang ${ }^{8}$, Y.L. Zhang ${ }^{1,3}$, Yi Zhang ${ }^{1,13}$, Yong Zhang ${ }^{1,3}$, B. Zhao $^{8}$, J. Zhao ${ }^{1,3}$, L. Zhao ${ }^{6,7}$, L.Z. Zhao ${ }^{14}$, S.P. Zhao ${ }^{13,22}$, F. Zheng ${ }^{33}$, Y. Zheng ${ }^{8}$, B. Zhou ${ }^{1,3}$, H. Zhou ${ }^{28}$, J.N. Zhou ${ }^{15}$, P. Zhou ${ }^{10}$, R. Zhou ${ }^{9}$, X.X. Zhou ${ }^{8}$, C.G. Zhu ${ }^{22}$, F.R. Zhu ${ }^{8}$, H. Zhu ${ }^{17}$, K.J. Zhu ${ }^{1,2,3,6}$ and X. Zuo ${ }^{1,3}$

${ }^{1}$ Key Laboratory of Particle Astrophyics \& Experimental Physics Division \& Computing Center, Institute of High Energy Physics, Chinese Academy of Sciences, 100049 Beijing, China.

${ }^{2}$ University of Chinese Academy of Sciences, 100049 Beijing, China.

${ }^{3}$ TIANFU Cosmic Ray Research Center, Chengdu, Sichuan, China.

${ }^{4}$ Dublin Institute for Advanced Studies, 31 Fitzwilliam Place, 2 Dublin, Ireland.

${ }^{5}$ Max-Planck-Institut for Nuclear Physics, P.O. Box 103980, 69029 Heidelberg, Germany.

${ }^{6}$ State Key Laboratory of Particle Detection and Electronics, China.

${ }^{7}$ University of Science and Technology of China, 230026 Hefei, Anhui, China.

${ }^{8}$ School of Physical Science and Technology \& School of Information Science and Technology, Southwest Jiaotong University, 610031 Chengdu, Sichuan, China.

${ }^{9}$ College of Physics, Sichuan University, 610065 Chengdu, Sichuan, China.

${ }^{10}$ School of Astronomy and Space Science, Nanjing University, 210023 Nanjing, Jiangsu, China.

${ }^{11}$ Center for Astrophysics, Guangzhou University, 510006 Guangzhou, Guangdong, China.

${ }^{12}$ School of Physics and Technology, Wuhan University, 430072 Wuhan, Hubei, China.

${ }^{13}$ Key Laboratory of Dark Matter and Space Astronomy, Purple Mountain Observatory, Chinese Academy of Sciences, 210023 Nanjing, Jiangsu, China.

${ }^{14}$ Hebei Normal University, 050024 Shijiazhuang, Hebei, China.

${ }^{15}$ Key Laboratory for Research in Galaxies and Cosmology, Shanghai Astronomical Observatory, Chinese Academy of Sciences, 200030 Shanghai, China.

${ }^{16}$ Key Laboratory of Cosmic Rays (Tibet University), Ministry of Education, 850000 Lhasa, Tibet, China.

${ }^{17}$ National Astronomical Observatories, Chinese Academy of Sciences, 100101 Beijing, China.

${ }^{18}$ School of Physics and Astronomy \& School of Physics (Guangzhou), Sun Yat-sen University, 519000 Zhuhai, Guangdong, China.

${ }^{19}$ Dipartimento di Fisica dell'Università di Napoli 'Federico II”, Complesso Universitario di Monte Sant’Angelo, via Cinthia, 80126 Napoli, Italy.

${ }^{20}$ School of Physics and Astronomy, Yunnan University, 650091 Kunming, Yunnan, China.

${ }^{21}$ D'epartement de Physique Nucl'eaire et Corpusculaire, Facult'e de Sciences, Universit'e de Gen 'eve, 24 Quai Ernest Ansermet, 1211

Geneva, Switzerland.

${ }^{22}$ Institute of Frontier and Interdisciplinary Science, Shandong University, 266237 Qingdao, Shandong, China.

${ }^{23}$ Department of Engineering Physics, Tsinghua University, 100084 Beijing, China.

${ }^{24}$ School of Physics and Microelectronics, Zhengzhou University, 450001 Zhengzhou, Henan, China.

${ }^{25}$ Institute for Nuclear Research of Russian Academy of Sciences, 117312 Moscow, Russia. 
${ }^{26}$ School of Physics, Peking University, 100871 Beijing, China.

${ }^{27}$ School of Physical Science and Technology, Guangxi University, 530004 Nanning, Guangxi, China.

${ }^{28}$ Tsung-Dao Lee Institute \& School of Physics and Astronomy, Shanghai Jiao Tong University, 200240 Shanghai, China.

${ }^{29}$ Yunnan Observatories, Chinese Academy of Sciences, 650216 Kunming, Yunnan, China.

${ }^{30}$ Department of Physics, Faculty of Science, Mahidol University, 10400 Bangkok, Thailand.

${ }^{31}$ Moscow Institute of Physics and Technology, 141700 Moscow, Russia.

${ }^{32}$ Key Laboratory of Radio Astronomy, Purple Mountain Observatory, Chinese Academy of Sciences, 210023 Nanjing, Jiangsu, China.

${ }^{33}$ National Space Science Center, Chinese Academy of Sciences, 100190 Beijing, China. 